\title{
Hybrid Conferences in the New Normal: Reality in a resource restricted context.
}

\author{
Karunathilake, I.M. ${ }^{1,3}$, Perera, B.J.C. ${ }^{3}$, Amarakoon, P.M. ${ }^{2,3}$, Ihsan, F.R. ${ }^{2,3}$, Mudiyanse, R.M. ${ }^{3}$, \\ Manawadu, M.C.K. ${ }^{1}$, Chandrasena, U. ${ }^{1}$, Wijewickrama, A. ${ }^{5}$, Silva, L.H.S.C. ${ }^{6}$, Peiris, J.S.M. ${ }^{7}$, \\ Low, W. $Y^{4}$, de Abrew, $A^{1}$
}

\section{Introduction}

The year 2020 was unprecedented, with COVID-19 inflicting an unexpected degree of damage and uncertainty on humanity. During such circumstances, professional organizations have the responsibility of providing accurate and up to date information on the global and local situation, as well as on prevention and management of COVID-19. For many professional organizations and institutes of higher learning, scientific conferences are the main platform for sharing and disseminating information.

Due to COVID-19 restrictions, many organizations opted for fully virtual conferences. While virtual conferences have the potential to broaden participation and strengthen focus on technical content, they face serious challenges in promoting social interactions and broadening the scope of discussions.

A hybrid conference combines a "live" inperson activity with a "virtual" online component. It should promote attendee engagement with networking while mainlining high technical quality and adherence to COVID19 preventive measures (Sox et al., 2017; Sá et al., 2019).

${ }^{1}$ Faculty of Medicine, University of Colombo, Sri Lanka

${ }^{2}$ Postgraduate Institute of Medicine, University of

Colombo, Sri Lanka

${ }^{3}$ Sri Lanka Medical Association

${ }^{4}$ President, Asia Pacific Academic Consortium for Public Health (APACPH)

${ }^{5}$ Chief Consultant Physician, National Institute of Infectious Diseases, Sri Lanka

${ }^{6}$ National Operational Centre for Prevention of COVID 19, Sri Lanka and Commander of Sri Lanka Army

${ }^{7}$ National University of Hong Kong

Corresponding author: Dr. Rizka Ihsan

rizka_ihsan@pgim.cmb.ac.lk
In this article, we discuss our experience in organizing a series of hybrid conferences, which, to the best of our knowledge, were the first hybrid academic conferences on public health and medical education in South East Asia. Since then, other conferences followed, and discussions emerged in the research community about hybrid conferences becoming the norm in the future. Yet for all that, little is known about how to design hybrid conferences and how they impact on the participants' experience.

Given the growing interest in hybrid conferences, we aim to provide insight on organizing similar events in the future and to inform and inspire an on-going conversation around how the networking research community can leverage the opportunities offered by virtual conferencing while minimizing the drawbacks on social interactions.

\section{Our initial experience in conducting hybrid conferences}

In early March 2020, the news about the COVID-19 outbreak became increasingly alarming, despite the ongoing restrictions on international and domestic travel. As the total number of confirmed cases rose to 66, the Sri Lankan state imposed a nationwide lockdown on March 20, escalating efforts to slow down the transmission of the disease (Department of Government Information, 2020).

The need for timely and accurate scientific information was greatly felt. Taking into consideration this global concern, the possibility of combining a "live" in person event with a "virtual" online component, the proposed Hybrid format, was identified. The first activity of such a nature was proposed as a collaborative activity jointly organized by the Asia Pacific Academic Consortium of Public Health (APACPH) and the Sri Lanka Medical Association (SLMA). In the absence of definitive scientific knowledge, we had only our 
intuition to rely on as the best course of action for hosting this event, together with our previous experience in conducting a series of webinars on COVID-19.

\section{The Asia Pacific Academic Consortium of} Public Health (APACPH) international webinar on COVID-19, held in collaboration with the Sri Lanka Medical Association (SLMA), titled "Breaking the transmission chain through community empowerment" took place on the 30th of April 2020. This was a timely meeting at a point when the Asia Pacific region was facing the initial onslaught of the pandemic. The event was chaired by the Presidents of APACPH and SLMA and included resource personnel from several countries in the Asia Pacific including Sri Lanka, Australia, China, Hong Kong, Malaysia and Singapore. It was felt that this webinar had to be conducted quickly in order for us to learn and share experiences and best practices among APACPH member institutions within the region in tackling this pandemic. (SLMA, 2020).

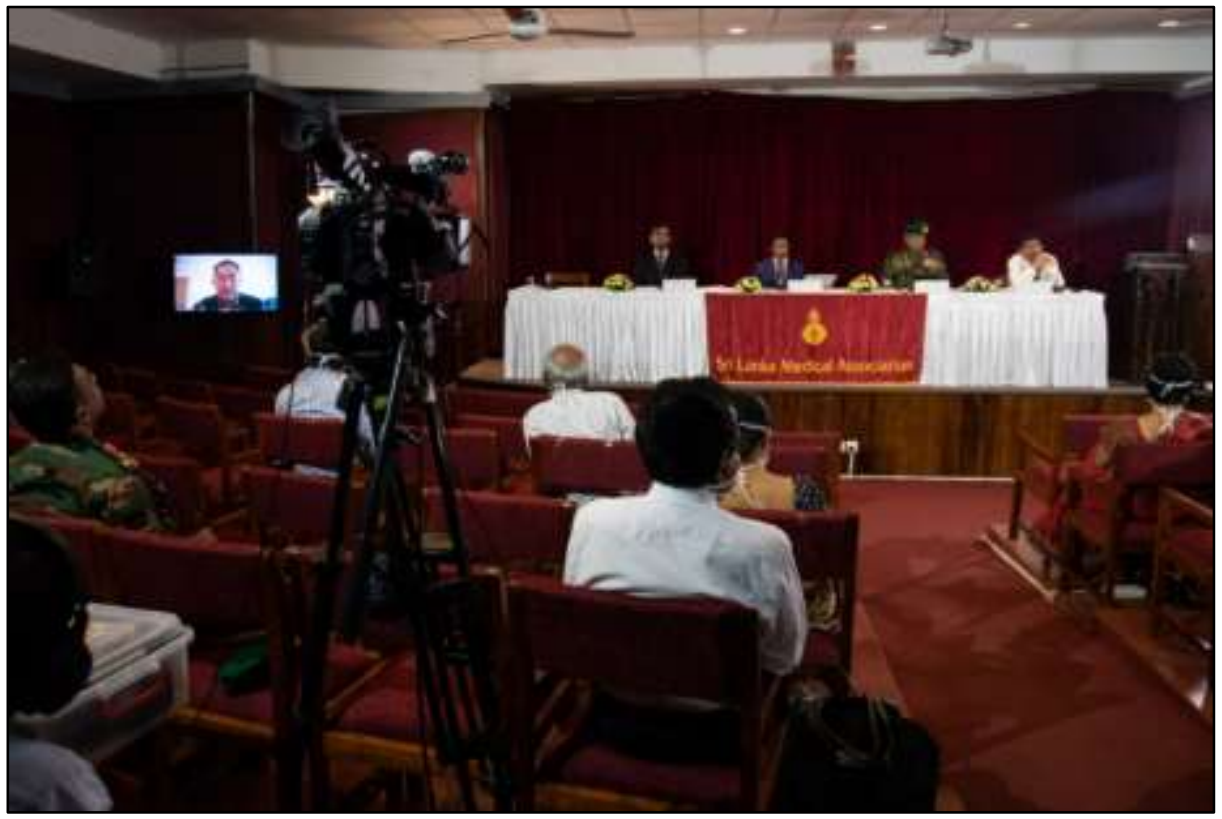

Figure 1: First joint hybrid meeting jointly organized by SLMA and APACPH

Colombo Medical Faculty Conference: Based on the success of this initial experience, the SLMA, together with the Faculty of Medicine, University of Colombo, co-hosted a Hybrid International Conference in June 2020. 1st June 2020, marked the Sesquicentennial Anniversary of the Faculty of Medicine, University of Colombo. It was a historic occasion commemorated in a fitting manner by staff, students and alumni of the Faculty featuring an in-studio audience of distinguished alumni and over 600 virtual participants from 17 different countries. Speakers included alumni from Australia, Bhutan, Canada, Singapore, UK, USA as well as the World Health Organisation.

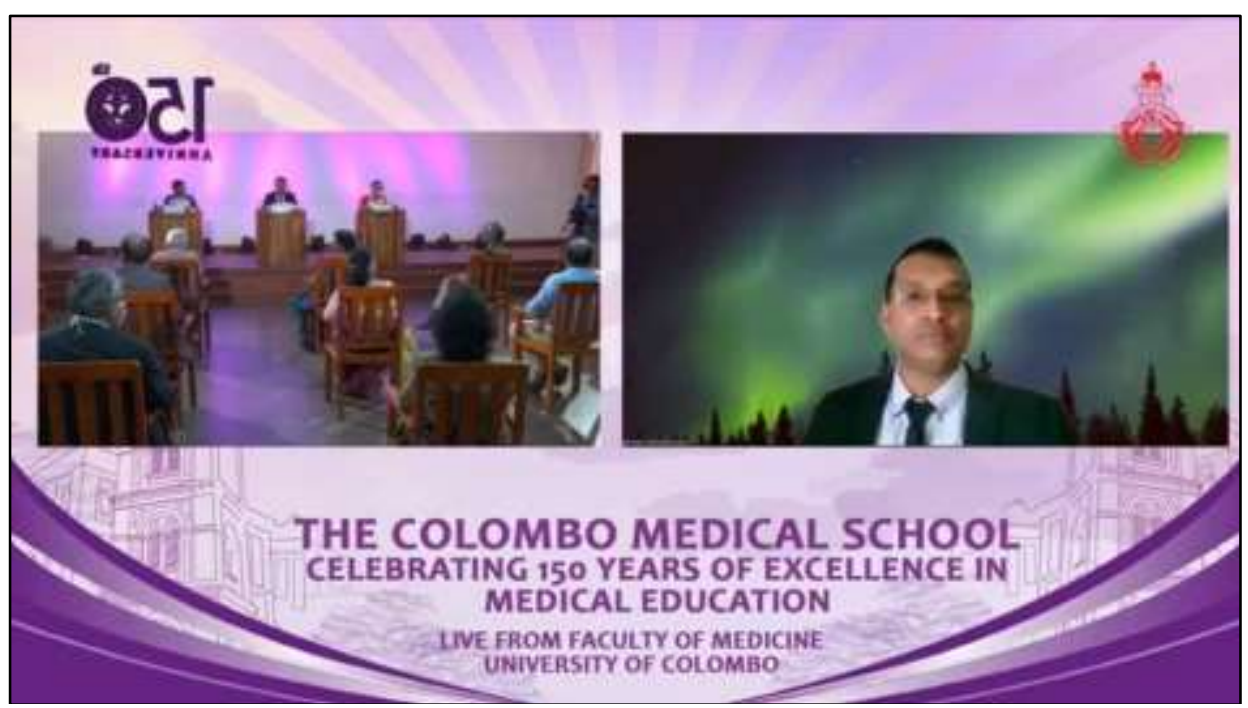

Figure 2: Virtual and in-person participants connected using multiple platforms 
The third experience was the $133^{\text {rd }}$ Anniversary International Medical Congress of the SLMA, which was held as a hybrid conference from $24^{\text {th }}$ to $26^{\text {th }}$ July 2020 . An aptly themed Congress, "Professional Development for Quality Enhancement of Healthcare: Beyond the COVID-19 pandemic". With over 700 participants joining online. It was a complete conference conducted over three days in two parallel venues with all the regular components including the inauguration ceremony, a keynote address, six guestlectures, eight symposia, two plenaries and culminating with the Doctors' Concert. This was a unique blend of tradition and technology in the era of new normal, combining the best of reallife and virtual. The success of the 133rd Anniversary International Medical Congress of the SLMA was an eye opener demonstrating the potential of technology in providing Continuous Professional Development (CPD) in Sri Lanka (SLMA, 2020).

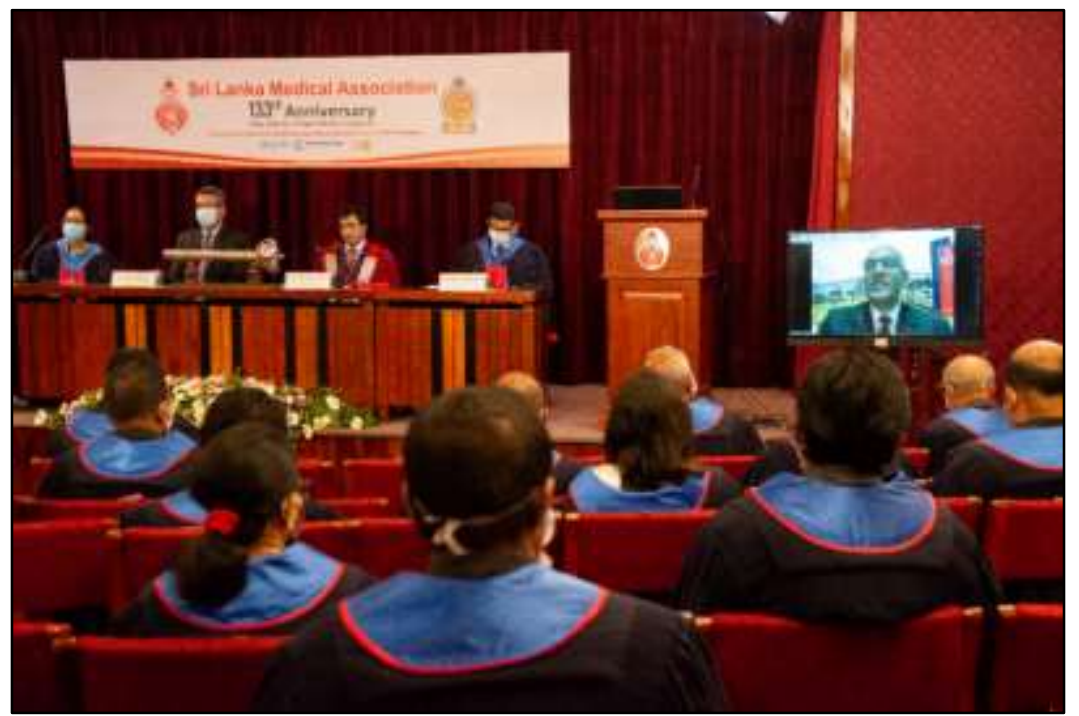

Figure 3: The Anniversary Medical Congress of SLMA conducted as a virtual event

Building on these experiences, the annual conference of Asia Pacific Academic Consortium for Public Health (APACPH) was held from 5th to 9th December 2020. SLMA in collaboration with the Faculty of Medicine, University of Colombo, were the co-hosts of the APACPH 2020 conference. The main conference of APACPH was held on 8th and 9th December 2020, with limited in-person attendance and hundreds of participants from over 40-member countries joining virtually. The enthusiastic participation of overseas researchers and internationally reputed experts in public health was the highlight, as, despite the pandemic, they were able to join the proceedings virtually.

The ceremonial inauguration was a hybrid event and shared unique aspects of Sri Lankan culture with the international participants. Toplevel artistes and media personalities were invited to perform during the inauguration as well as in interludes during the conference. All performances were planned to convey messages related to COVID-19.

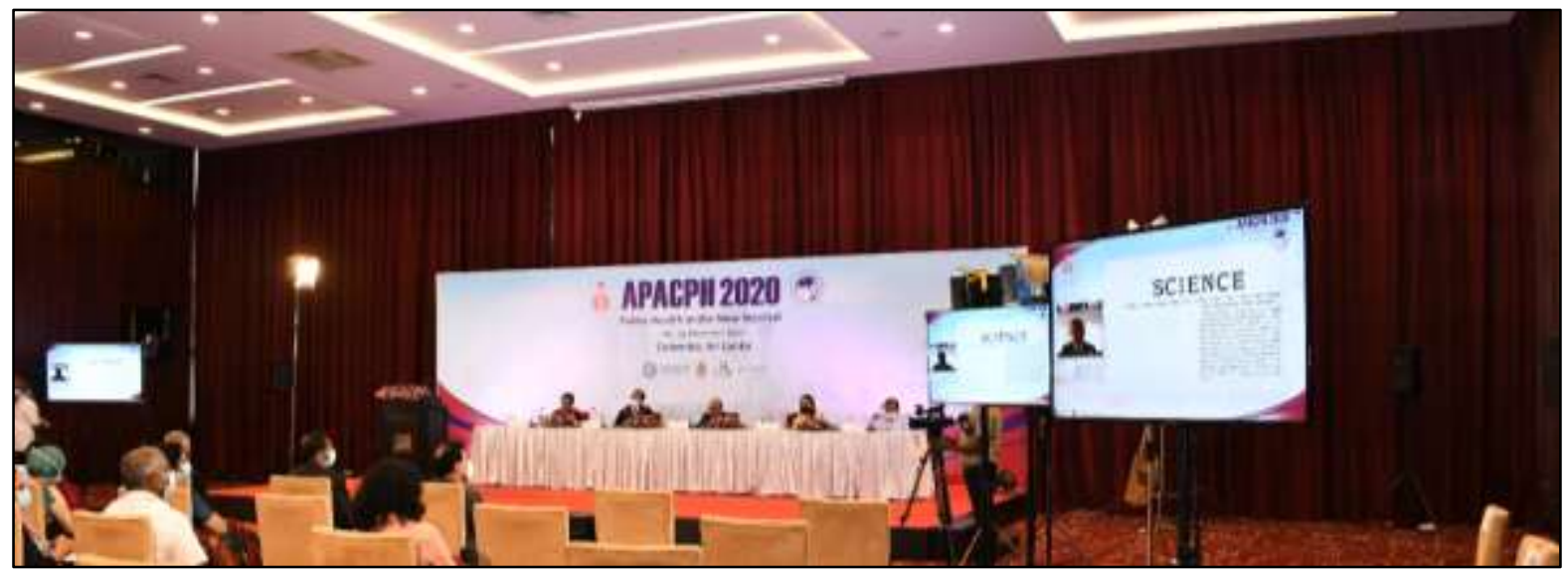

Figure 4: APACPH 2020 Hybrid Conference 


\section{What have we learnt about organizing Hybrid Conferences?}

The Hybrid format was planned to make the experience accessible and useful for participants and presenters while minimizing the risks associated with technical, social, and other factors. Based on our experience, we present 12 golden rules to make a hybrid conference a reality:

Rule 1: Identify the "Dream team"

Rule 2: Establish communication early

Rule 3: Identify Technological Requirements

Rule 4: Design the Conference Programme

Rule 5: Identify the Venue

Rule 6: Advertise aggressively

Rule 7: Plan the Budget

Rule 8: Enlist Financial Support

Rule 9: Execute Pre-conference Preparations

Rule 10: Prepare for any technical difficulties

or breakdowns

Rule 11: Plan coordination during the

Conference with military precision

Rule 12: Follow up with enthusiasm

\section{Rule 1: Identify the "Dream team"}

In a culture that was sceptical about the effectiveness of virtual platforms in education prior to the APACPH international webinar on COVID-19, identification of colleagues and graduate students to serve on conference committees required meticulous consideration. In addition to general aspects considered in face-to-face conferences such as perseverance, expertise and dedication, flexibility, technological familiarity, as well as eagerness to learn, optimism and out-of-the box thinking are traits that were favoured.

Due to the COVID-19 related restrictions of physical gatherings, technology had to be carefully utilized with a multi-talented team of experts. Organizing such a team at short notice was facilitated by the long-standing networking and cordial relationships established over the years by the SLMA. Mentoring and transfer of knowledge also took place during these events to ensure sustainability of technical competencies and proliferation of human resources to cater to the growing demands.

It would be useful to set up subcommittees such as technical committees, fund-raising committees, and scientific committees, among others, to deal with conference requirements as they arise. Although the titles of these subcommittees are identical to those of any conference the roles are significantly different.

\section{Rule 2: Establish communication early}

One of the main challenges was to coordinate with the different organizations, resource persons and all other stakeholders during a time where everyone was overwhelmed with responsibilities regarding containing the COVID-19 pandemic. Establishing early communication was the key to success.

One challenge related to conducting hybrid conferences is the task of maintaining communication between the organizers, participants and the presenters. Within subcommittees, individuals were assigned the duty of maintaining frequent communication with relevant stakeholders whereas a designated team under the supervision of a steering committee member was set up where each member was responsible for all communication of an agenda item. This structured and methodical plan for communication proved efficient to meet the rising demand of constant communication by means of email, telephone conversations and online meetings as well as reminders prior to the event as opposed to the more familiar faceto-face conference. This not only enhanced the participation but also contributed to maintaining the timing of the session, smooth execution as well as enhancing the participant experience.

Details of the agenda, timing and platform utilization were communicated to keynote and invited speakers in advance. These resource persons were given allocated time, at their convenience, to familiarize with the designated software and clarify technical queries, three weeks prior to the conference.

\section{Rule 3: Identify Technological Requirements}

The online platform selected for hybrid conferences needs to fulfil several key requirements. It is essential that the platform is able to seamlessly accommodate multiple speakers joining virtually from many parts of the world. We opted for the Zoom VideoConferencing Platform, since it offered a number of additional advantages such as providing a chat feature for private and public messages, offering the ability to share screens and playback pre-recorded videos, supporting the ability to record and store sessions, accommodating multi-platform support, including mobile phones (https://zoom.us/). In addition, we used the advanced features in the Zoom Platform for integration with the existing registration portal of SLMA so that participants 
received dedicated zoom links upon registration and completion of payment. The Application Programme Interface (API) of the Zoom Platform was utilized by the technical team for bulk registration of users, minimizing the manual workload with registration, which we might have had to deal with otherwise.

Conducting a virtual conference requires a blend of technologies other than the video conferencing platform. These include lighting and audio system, video capture and recording, video editing and mixing with video conferencing platform, as well as web and social media technology for promotion and engagement with participants. The technologies per se do not materialise without technical competency of human resources.

Online platforms are usually criticized for the difficulty of sustaining engagement of the participants for long durations. Conducting of poster sessions was backed by the innovative idea of bringing in the experience of physical conference to the virtual experience. Our inhouse team designed an online platform which provided the participants the facility to view the posters in a three-dimensional virtual environment of a conference hall in which they can roam within the hall viewing a series of posters. This was a low-cost solution designed on common web development frameworks within a short span of time and then integrated to the conference web portal to provide a seamless experience. This brings about a hint of gamification to a more academic event, which ultimately makes for a richer user experience. This can be further highlighted as a classical case of application of technology to materialize innovative ideas which emerged from within the team.

\section{Rule 4: Design the Conference Programme}

The transition to a hybrid format required meticulous planning of the programme following careful consideration of factors such as optimal resource management,-participant engagement, feasibility and, maintaining energy as well as enthusiasm. Academic networking, presenting research, following presentations, gaining new knowledge and participating in meaningful dialogue for professional development are participant goals that had to be kept in mind while designing. The organizing committee was tasked with including all key elements of a face-to-face conference on a simple, user-friendly interface while adhering to public health measures and providing an enjoyable, effective and positive experience for both participants and resource persons. Furthermore, the prospect of spending long hours in front of a screen, having alternatives for sudden technical glitches, accommodating different time-zones of all presenters and resource persons as well as deciding on a minimal number of on-site participants for smooth execution of the conference were challenges the organizing committee faced, quiet successfully.

The International Medical Congress of the SLMA and APACPH attract many international participants. An advantage of virtual conferences is the flexibility for participants to attend an array of sessions of their choice, in multiple events that take place simultaneously. However, addressing time zone differences is vital. While it was impossible to identify time slots convenient to all participants, the organizing committee considered the geographical locations of resource persons and registered participants (Misa et al, 2020).

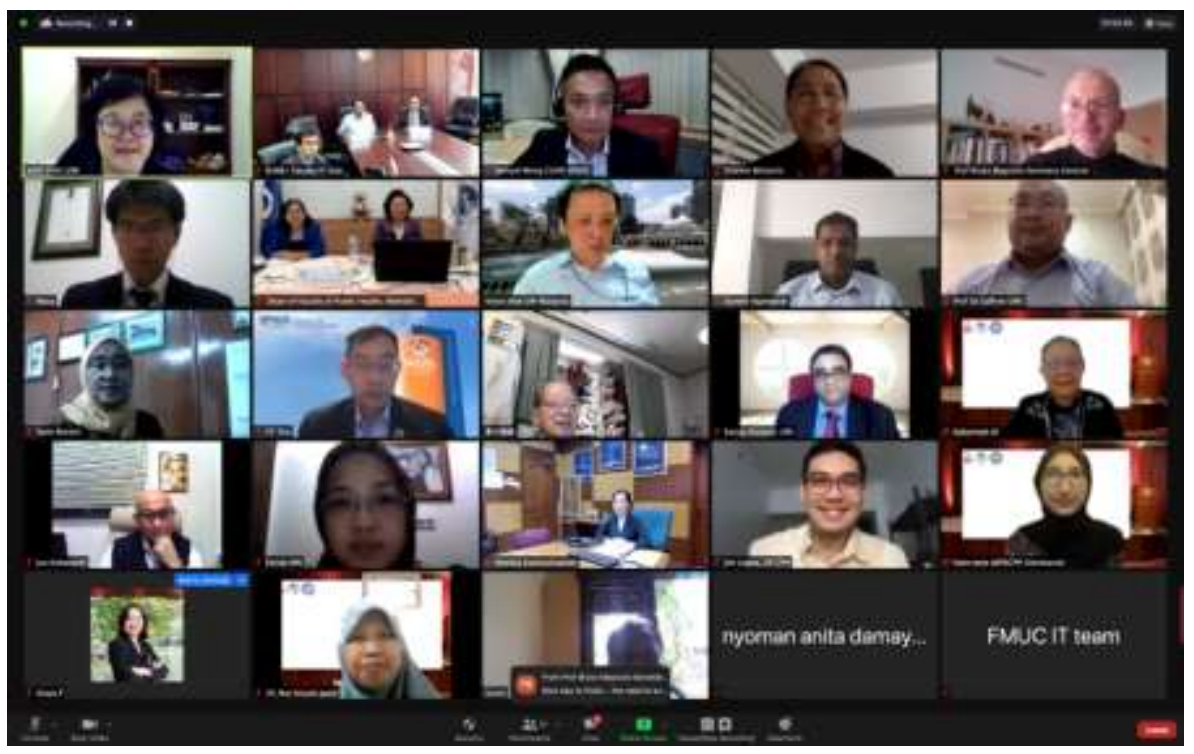

Figure 5:APACPH 2020-participants representing several countries 
Virtual participants are easily distracted by their surroundings and do not enjoy spending long hours in front of a screen. Therefore, all agenda items were limited to a maximum of 1-2 hours and considerable breaks were incorporated in between to provide flexibility and time to refresh. Execution of a hybrid conference is a resource intense process. Conducting live parallel symposia require duplication of technical facilities that consume immense resources and finances. Hence, the number of parallel symposia conducted were very limited.

In addition to multiple measures to enhance participant engagement, the resource persons were encouraged and facilitated to include live polls in their sessions and utilize interactive presentation software such as Mentimeter. Conference activities such as the photography competition were held on a virtual 3D platform to provide a real-life experience.

\section{Rule 5: Identify the Venue}

The venue should fulfil multiple requirements, combining the technical needs for physical as well as virtual meetings. Creating the ambiance for an international conference, logistics, catering etc, are as important as high-speed broadband internet and audio-visual and back up facilities.

When such facilities were not available, the only option was to institute makeshift arrangements. However, low-cost solutions are widely available to establish makeshift arrangements. Application of virtual backgrounds is no-longer a rare and expensive feature. Most video conferencing platforms are equipped with virtual backgrounds as well as automatic lighting adjustments to capture better quality videos in low-light conditions. Modern codecs and compression technologies have minimized the streaming bandwidth while ensuring audiovideo quality. Software is available to produce studio quality recordings without the requirement of high-quality video production hardware. Investments should be made on procuring up-to-date video production equipment as well as building capacity within your team. However, much of the required knowledge is now freely available as online tutorials, documents and other resources.

\section{Rule 6: Advertise aggressively}

The APACPH 2020 Conference was organized in less than two months. Calls for abstract submission and advertising for the photography competition was promoted via the apacph.org website, SLMA website, the SLMA Facebook page, as well as emails to colleagues in the field. Word- of- mouth advertising then helped us reach a vast audience both locally and internationally. Special concessions for the conference registration fee were provided to participants from low-income countries and for university students. The collected funds were used for the costs incurred to organise and conduct the conference.

\section{Rule 7: Budget reflecting the hybrid nature}

A significant proportion of expenses in an onsite in-person conference are dedicated to refreshments and provision of facilities for onsite participants. In a hybrid conference this cost is significantly less while providing more opportunity for a wider audience to join. This creates an impression in the minds of many that virtual conferences do not require financial support due to absence of costs for conventional logistics related expenses. It is prudent to understand that the quality of digital content delivered is the single-most important factor for engagement of online participants. To ensure that, investments are required for costs related to video recording, audio, lighting and high-quality digital production. This is a crucial factor, often missed by many, when planning virtual conferences.

\section{Rule 8: Enlist Financial Support}

Organizing hybrid conferences is resource
intensive and expensive. Securing sponsorships is a daunting challenge during a pandemic. Innovative approaches are required to convince potential sponsors regarding the benefits of this novel approach. We made sure that our sponsors and the host organization were properly acknowledged, as we took the following additional steps. Posters and banners with sponsor logos were displayed at the conference venue. The sponsors provided promotional videos which were inserted between presentations and during breaks. The size and diversity of the potential audience was an added incentive.

The recordings of pre-conference webinars and workshops were edited and uploaded to YouTube. They were also emailed to participants in order to garner publicity to the main conference. Other social media platforms such as Facebook, Twitter and academic WhatsApp groups were constantly updated with conference related information to remind registrants and attract more participants. 


\section{Rule 9: Execute Pre-conference Preparations}

With prior consent, it was decided to pre-record all oral presentations for several reasons including mitigative alternatives to network glitches during presentations and development of digital resources. A common template for oral presentations and detailed instructions including allocated time slots for recordings, were sent via email to all presenters in advance. Recording was done in two sessions via zoom to maintain quality, duration and uniformity across presentations. A designated committee member was present and tasked with the responsibility of creating presentation videos and assisting presenters with minor issues.

Recorded presentations were used only when absolutely necessary, mainly for Free Paper Presentations. It was not used for invited keynote speeches, since a recorded presentation would deprive participants of the opportunity to interact with the speaker during the presentation.

\section{Rule 10: Prepare for any technical difficulties or breakdowns}

All PowerPoint presentations of speakers were obtained in advance and stored methodically, enabling quick retrieval in the event of a technical malfunction. These media files were also copied to another shared storage portal, so that general chairs could play the videos in case the presentation chair became unavailable during the conference. Pre-recorded oral presentations proved very useful in the event of a network malfunction. Furthermore, multiple servers were utilized as backup in case of a failure during the conference.

Dedicated human resources, both onsite and offsite were assigned to undertake the task of editing videos of all recorded sessions, to be uploaded on the conference YouTube channel shortly after the day's proceedings.

\section{Rule 11: Plan coordination during the Conference with military precision}

Deciding on the minimum number of on-site participants was quite challenging as this number included the technical team, support staff, resource persons, organizing committee members, distinguished guests and selected participants. The head of each team decided on the minimum number of individuals required to be physically present at the conference location for smooth execution of the conference. This ensured essential technical, support staff and organizing committee were physically present. Further, a virtual support station was set up at the SLMA office premises to reduce the gathering at the location of the main conference. Keynote speakers, distinguished guests and invited speakers who preferred to be present in-person were welcomed. All other participants were requested to join the conference online. The participants had the opportunity to raise questions at the end of presentations.

The hybrid nature of all the conferences required coordination between the session chair and the audio-visual team. The day's programme was pre-planned and discussed. Team members ensured that speakers for the session had joined prior to the commencement and stayed logged in throughout. The speakers were given feedback on audio-video aspects such as microphone volume. Designated teams responsible for smooth execution of each agenda item had contact details of all resource persons to communicate any sudden changes and delays. This proved to be immensely useful in the event of a technical error during their sessions. It was well noted that the resource persons chairing the sessions needed to have reasonable competence in managing the virtual platform and the ability to actively monitor the engagement of participants and enhance interactivity.

\section{Rule 12: Follow up with enthusiasm!}

Objective of any scientific conference is to share knowledge and therefore, plan for dissemination of knowledge during follow up were instituted. We linked all content produced for the conference to the technical program web page. This includes a copy of the papers hosted at the respective authors' web sites, presentation slides, and links to the presentation videos hosted on YouTube. We explicitly requested presenters' consent before publicly posting their videos on YouTube. After the conference, we converted and placed all the videos on YouTube, and linked them to the technical programme

\section{Conclusion}

Hybrid conferences can be optimised to combine the best of both worlds, virtual and physical. However, the amount of planning, preparation and technological expertise required should not be underestimated. 
The most feasible and cost-effective option with the required features for our hybrid conferences was Zoom, although it carried the disadvantage of not being able to accommodate participants from countries such as China.

Interactive sessions and additional activities were incorporated to enhance academic networking, a goal of many conference participants. However, the degree of networking seen during in-person conferences could not be achieved to its fullest extent.

\section{Acknowledgments:}

Ministry of Health, Sri Lanka, WHO country office, Sri Lanka, World Bank country office, Sri Lanka, Council and Staff of the Sri Lanka Medical Association, Executive Committee of the Asia Pacific Academic Consortium for Public Health (APACPH), National Operational Centre for Prevention of COVID-19, for logistical and technological support. Dr Dinil Abeygunawardane and staff of the Institute of Multimedia Education, who provided the technological support. Dr Chiranthi Kongala Liyanage, Ms Akalanka Hettihewa, Ms Zainab Usuf, Ms Rishmika Peiris contributed during planning and implementation. Mr Ravibnadu VIdyapathi, Mr. Jagath Wickrmasinghe, Dr Nilanka Wickramasinghe Ms Michelle Dilhara and teams of artistes. Prof. Vajira Dissanayake, Dean and office staff of the Faculty of Medicine, University of Colombo.

\section{References}

APACPH (2020). Public Health in the New Normal. Abstracts and Proceedings of the Virtual Conference webcast live from Colombo, Sri Lanka, $5^{\text {th }}-9^{\text {th }}$ December 2020.

Department of Government Information (2020) Press Release 2020.03.20. Island-wide curfew notification. Government of Sri Lanka.

Gichora, N., Fatumo, S., Ngara, M., Chelbat, N., Ramdayal, K., Opap, K., Siwo, G., Adebiyi, M., El Gonnouni, A., Zofou, D., Maurady, A., Adebiyi, E., de Villiers, E., Masiga, D., Bizzaro, J., Suravajhala, P., Ommeh, S. \& Hide, W. (2010). Ten Simple Rules for Organizing a Virtual Conference-Anywhere. PLoS Computational Biology, 6(2), p.e1000650.

Misa, C., Guse, D., Hohlfeld, O., Durairajan, R., Sperotto, A., Dainotti, A. \& Rejaie, R., 2020. Lessons learned organizing the PAM 2020 virtual conference. ACM SIGCOMM Computer Communication Review, 50(3), pp. 46-54.

Sá, MJ, Ferreira, CM \& Sandro. S. (2019). Virtual and Face-To-Face Academic Conferences: Comparison and Potentials. Journal of Educational and Social Research. 9, pp. 35-47

SLMA (2020). Annual Report. [online] Sri Lanka Medical Association. Available at: <https:// slma.lk/publications/sIma-annual reports/>.

Sox, C. B., Kline, S. F., Crews, T. B., Strick, S. K., \& Campbell, J. M. (2017). Virtual and Hybrid Meetings: A Mixed Research Synthesis of 20022012 Research. Journal of Hospitality \& Tourism Research, 41(8), pp. 945-984.

Zoom Video Communications (2021). Video Conferencing, Cloud Phone, Webinars, Chat, Virtual Events | Zoom. [online] Available at: $<$ https://zoom.us/> [Accessed 8 July 2021]. 